Proceeding of the $1^{\text {st }}$ ICEENG conference, 24-26 March, 1998.

MILITARY TECHNICAL COLLEGE CAIRO-EGYPT

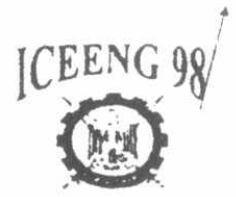

\title{
AUTOMATIC MODULATION RECOGNITION: A SURVEY AND OPEN ISSUES
}

\author{
Elsayed. E. Axzouz" and Eweda I. Eweda ${ }^{*}$
}

\begin{abstract}
This paper provides a tutorial review of modulation recognition techniques. The available modulation recognizers are classified into three main categories. The first category is concerned with the analog modulations only. The second category is concerned with the digital modulations only. The third category is concerned ivith both analog and digital modulation without any prior information about the nature of a signal. Comparisons between the available modulation recognizers in each category are devcloped. Sume of these modulation recognizers utilize the decision-theoretic approach, some utilize the pattern recognition, some combine both approaches and the rest utilize the ANNs approach.
\end{abstract}

\section{Introduction}

Signals travelling in space with diffcrent modulation types and different frequencies fall in a very wide band. Usually, it is required to identify and monitor these signals either for military or for civilian purposes. Civilian purposes are such as signal confirmation, interference identification and spectrum management. Civilian authorities may wish to monitor their transmissions in order to maintain a control over these activities as wall as detecting and monitoring the non-licensed transmitters. Military purposes are such as elcctronic warfare, surveillance and threat analysis. In electronic warfare applications, electronic support measures system plays an important role as a source of information required to cunduct elestronic counter measures, threat detection, ivarning, target acquisition and homing. Generally, any surveillance system in COMINT applications consists of three main blocks: recciver front-end (activity detection and frequency down conversion), modulation recognizer (key fealures extraction and classification) and output stage (normal demodulation and information exticction). At the output stage there are several functions performed and they are mainly related to informstior. extraction, recording and exploitations. All these functions are preceded by signal dencd:a!ntion. The prior information required for any modulation recognizer is the signal bandwidth, which can be determined in the receiver front-end

\footnotetext{
* Department of Electronic and Electrical Engineering, Military Technical College, Cairo, Egypt.
} 
stage. The information obtained from the receiver front-end, modulation recognizer and parameters estimator such as carrier frequency, signal bandwidth, modulation type, symbol rate, ... etc. are gathered to perform the signal demodulation and information extraction.

Generally, there are three philosophies for approaching the modulation recognition process in the available references namely 1) a decision-theoretic approach, 2) a statistical pattern recognition approach and 3) an artificial neural networks (ANNs) approach. In the decision-theoretic approach, probabilistic and hypotheses testing arguments are employed to formulate the modulation recognition problem. In the statistical pattern recognition approach, the classification system is divided into two subsystems. The first is a features extraction subsystem whose function is to extract the pre-defined features from the received data. The second is a pattern recognition subsystem, whose function is to indicate the modulation type of a signal. The work with pattern recognition comprises two phases - 1) a training phase to adjust the classifier structure and 2) a test phase that gives the classification decision. The work with ANNs approach comprises three main steps: 1) pre-processing and key features extraction, 2) a training phase to choose the best networks structure; e.g. determine the weights and biases of the chosen networks, and 3) a test phase, in which the performance evaluation of the chosen network is determined. The modulation recognizers, in the available references, were developed according to any of these approaches. There are also some recognizers combining these approaches. Also, there are five techniques for solving the modulation recognition problem. These are: 1) spectral processing, 2) instantaneous amplitude, phase, and frequency parameters, 3) instantaneous amplitude, phase, and frequency histograms, 4) combination of the previous three techniques and 5) universal demodulators.

Modulation recognition brings together many aspects of communication theory such as signal detection, parameter estimation, channel identification and tracking. Furthermore, modulation recognition environment may vary between two extremes - from no significant noise in the best situation to a very noisy one with interference and fading. Moreover, there are many practical problems facing the modulation recognition process. Some of these problems are due to the radio communication channel and the intercept receiver. These problems are such as: multi-path fading, weak signal reception, signal distortion, frequency instability, interference from adjacent channels and signal selection. These problems should be solved in pre-processing stage for perfect modulation recognition. The other problems are due to the nature of the received signal. These problems are such as: the weak segment of a signal (carrier absent or reduced and the pauses in transmission of analog moriulation), lower SNR reception, and the transmission time and the speed of computation. The details for some of these problems are discussed in [24] and the suitable solutions are presente ${ }^{\prime}{ }^{\prime}$. Modulation recognition is extremely important for several reasons. First, application of a signisl to an improper demodulator may partially or completely damage the signal information conten.c. It is worth noting that any partial damage of the signal information content considerably confi,sses the following deciphering process which converts the demodulated message from its non-intelligible (ciphered) form to the intelligible (deciphered) one. Second, knowing the correct rrodulation type helps to recognize the threat and determine the suitable jamming waveform. A'so, modulation recognition is important for national security.

From th، modulation recognition point of view, there are many classifications for communication signals as shown in Fig. 1. The first classification is according to the signal information content. In this c.lassification any communication signal can be categorized as one of four categories: 1) ampl itude signal, 2) angle modulated signal, 3) combined signal and 4) CW (unmodulated) signal. Amplitude signal is; characterized by the useful information being in the signal envelope (or more presisely the instantaneous amplitede). Angle modulated signal is characterized by the useful 
Proceeding of the $1^{\text {st }}$ ICEENG conference, 24-26 March, 1998.

information being in the instantaneous phase or in the instantaneous frequency. Combined signnal is characterized by the useful information is comprised in both the instantar eous amplitude and the instantaneous phase or frequency. CW signal is characterized by the fact that no useful information is transmitted. The second classification is according to the signal spectrum symmetry around the carrier frequency. Generally, the RF signal spectrum is composed of a carrier component plus two sidebands but in some types one or two of these components may be lost or reduced. Thus, due to the existence of the sidebands, any communication signa can be categorized as one of two categories: 1) symmetric signal and 2) asymmetric signal. l'erfestly symmetric signal is characterized by the fact that the signal powers in the two silebands are equal. Asymmetric signal is characterized by different powers in the two silebands. The third classification is according to the nature of the modulating signal used. In thi; classification, any communication signal can be categorised as one of two categories: 1) analog nodulated signal in which the modulating signal is analog such as speech signal and 2) digitally modulated signa, n which the modulating signal is digital such as data signal. In the first classification, it is requ red. for any modulation recognizer to determine where the intercepted signal :ontains the useful information; that is to identify whether the information exists in the instantan zous amplitude, the instantaneous phase, the instantaneous frequency, or a combination of tham. In the second classification, it is necessary to measure the signal spectrum symmetry around its carrier frequency. In the third classification, it is desired to know what is the sourc $ə$ of the modulating signal.

This papers is concerned with reviewing the most recent papers (since 1@84) in the areil of modulation recognition. The available references [1]-[27] can be classified into three catego-ies according to the modulation types considered in each of them. The first category is concerned vith the recognition of analog modulations only (AMRAs) [4], [7], [15], [17], [20], [22], [25], [26] and [27]. The second category is concerned with the recognition of digital modulations only (DMR As) [1], [6], [10], [11], [13], [14], [16], [18], [19], [21], [23], [25], [26] and [27]. The third category is concerned with the recognition of both analog and digital modulations without any prior information (ADMRAs) [2], [3], [5], [8], [9], [12], [26], and [27]. It is worth no ing hat in the first category, none of the available references considered the VSB, and the con bined modulations except [22] and [25] - [27]. The oldest version of modulation recognizers [2] used a bank of demodulators, each used for only one type of modulation. An operator examining or listening, to the demodulators outputs could decide about the modulation type of the received signal. This

recognizer however requires long signal duration and highly skilled operator. The automatior of this recognizer is achieved by introducing a set of intelligence decision algorithms at the demodulators output as shown in Fig. 2. However, the implementation of this recognizer is complex and requires excessive computer storage. Moreover, the number of $\mathrm{mc}$ dulation types that can be recognized is based on the number of the demodulators used.

\section{Recognizers for Analog Modu!ated Signals Only (AMR Is)}

Fabrizi et. al. [4] suggested a modulation recognizer which utilizes the decision-theoretic approach for analog modulations, and it is based on the variations of bot 1 the instantaneous amplitude and the instantaneous frequency. The key features used are the ratio of the envelope peak to its mean, and the mean of the absolute value of the instantaneots frequency. This recognizer is used to discriminate betseen some types of analog modulatio $1-C W, A M, D S B$, SSB and FM. The performance eva! ation of this recognizer is derived from 24 realizations exich with 250 msec. length for each modc:ntion typ: of interest. In [4], it is claimed hat with these two 
key features the discrimination between the AM and the FM signals could be achieved at SNR > $35 \mathrm{~dB}$. However, SSB could be recognized from AM and FM signals at SNR $>5 \mathrm{~dB}$.

Chan and Gadbois [7] developed a modulation recognizer which utilizes the decision-theoretic approach, and it is based on the envelope characteristics of the intercepted signal. It uses the ratio $\mathbf{R}$ of the variance of the squared instantaneous amplitude to its mean square, as shown in Fig. 3 , as a decision criterion to decide about the correct modulation type. This recognizer is used for the recognition of some analog modulated signals - CW, AM, DSB, SSB and FM. The choice for the ratio $\mathrm{R}$ to discriminate between these modulation types is based on the following fact: in noisefree signals, the ratio $\mathrm{R}$ is ought to be zero for FM and CW signals and close to unity for AM signals. Also, the ratio R for DSB signal is ought to be 2 and it is equal to 1 for SSB signals. The simulation results for the developed recognizer were derived from 200 realizations for each modulation type of interest, each with length 2048 samples (equivalent to $20 \mathrm{msec}$.) [7, Table 3]. In [7] it is claimed that at $\mathrm{SNR}=7 \mathrm{~dB}$, the probability of correct modulation recognition is $100 \%$ for FM signals, $90.5 \%$ for AM signals, $80.0 \%$ for SSB signals and $94.0 \%$ for DSB signals. Furthermore, in [7] nothing is mentioned about the recognition of the VSB and the combined modulated signals. Moreover, since it depends on the envelope characteristics only, it cannot recognize the signals having both amplitude and phase information.

Nagy [15] proposed a modulation recognizer for analog modulations. In this recognizer, the Chan and Gadbois parameter [7], $\mathbf{R}$, in addition to the variance of the instantaneous frequency normalized to the squared sample time are used as key features to discriminate between the different modulation type of interest. The modulation types that can be classified by this recognizer are AM, DSB, SSB, FM, and CW. In [15], it is mentioned that the performance evaluation of this recognizer was derived from 500 realizations, each with $250 \mathrm{msec}$. length for each modulation type. In [15], it is claimed that the different modulation types have been classified with success rate $>90.0 \%$ at $\mathrm{SNR}=15 \mathrm{~dB}$ except the SSB $(66.0 \%$ success rate $)$. So, [15] developed another key feature, which is the mean value of the instantaneous frequency. In this case the SSB has been classified with success rate $>94.0 \%$ at SNR $=15 \mathrm{~dB}$, and the other types have been classified with success rate $100 \%$.

Jovanovic et. al. [17] introduced a modulation recognizer to distinguish between a pure carrier wave $(\mathrm{CW})$ and a low modulation depth $\mathrm{AM}$ in a noisy environment. The key feature used is the ratio of the variance of the in-phase component to that of the quadrature component of the complex envelope of a signal. The only thing that mentioned about the performance evaluation is that the proposed key feature is a highly reliable tool for separating the AM signals with low modulation depth from the unmodulated carrier even if the SNR is poor.

Al-Jalili [20] proposed a modulation recognizer to discriminate between the USB and LSB signals. This recognizer is based on the fact that the instantaneous frequency of the USB signal has more -ve frequency spikes than the +ve ones, and the opposite for the LSB signal. The key feature used in this recognizer is defined as the ratio, G, of the number of -ve spikes to the number of the $+v e$ ones of the instantaneous frequency. So, $G>1$ for USB and $\mathbf{G}<1$ for LSB. In [20] the performance measure is derived from 10 realizations, each with $128 \mathrm{msec}$. for each modulation type and it is claimed that this recognizer perform well for SNR $\geq 0 \mathrm{~dB}$.

Nandi and Azzouz [22] introduced a global procedure for modulation recognition of the well known analog modulation types. This recognizer utilizes the decision-theoretic approach. It comprises two main steps. First, the decision about the modulation type is taken first from each 
available segment. Second, a global decision is determined from all the available segments of the intercepted signal frame by comparing the global decision w th each segl ent decision. The decision from each segment is based on four key features. These are: 1) the maximum value of the spectral power density of the normalized-centered instantaneous amplituce, 2) the stanciard deviation of the absolute value of the centered non-linear component of the instantaneous phase in the non-weak intervals of a signal segment, 3) the standard deviation of the (lirect (not absolute) value of the centered non-linear component of the instantaneous phase, and 4) the RF spectrum symmetry measure around the carrier frequency of the intercepted signal. Th: way' for extracting these key features is as shown in Fig. 4. In this recognizer the decision about he modulation type is carried out according to the decision rules shown in Fig. 5. This rec ognizer is usec to discriminate among the AM, DSB, VSB, LSB, USB, FM, and combined modulated signals. In [22], all the modulation types of interest have been classified with success rate $>90.0 \%$ at the SNR of $10 \mathrm{~dB}$. Excluding the VSB, LSB, and USB, the success rate for all th other modulation types of interest is $>98.0 \%$. Furthermore, Azzouz ard Nandi [26] introducerl a modification for the decision flow of the algorithm shown in Fig. 5. In [26], $t$ is mentioned that, based on the aforementioned four key features, many algorithms can be developed acccrding to the chosen decision flow. It was found that for one the modified algorithms, the ov rall success rate is $99.4 \%$ at the SNR of $10 \mathrm{~dB}$, and it is $99.9 \%$ at the SNR of $20 \mathrm{~dB}$. In [22] and 26] due to the simplicity of both the key features extraction as shown in Fig. 4 and the deci ion rules as shown in [24; Figs. 2.3 and C.1-C.4] (simple logic functions), these algorithms can be used for on-line analysis. Moreover, Nandi and Azzouz proposed a modulation recognize - which utilizes the ANN approach [25] as shown in Fig. 6a. It is based on a single hidden layer ANN and the same data set used in [22]. It was found that all the modulation types of interest have been classified with success rate $>95.0 \%$. Excluding the USB, the success rate is $>98.0 \%$ at 1 ] dB SNR. In $[27$, Azzouz and Nandi introduced a double hidden layer ANN modulation recignition algoritl m. Using the same data set in [22] and [25], it was found that all the modulation types of interest have been classified with success rate $>98.0 \%$. The training and test procedu e of the ANNs are shown in Figs. 6 and 7 respectively.

\section{Recognizers for Digitally Modulated Signals Only (DMIRAs)}

Liedtke was the first to present the concept of modulation recognition applied to digital modulations. Liedtke [1] proposed a modulation recognizer for some types of cigital modulaticns - ASK2, FSK2, PSK2, PSK4, PSK8 and CW. This recognizer ut lizes the unis ersal demodularor technique. The key features used to discriminate between these types are the amplitude histogra $n$. the frequency histogram, the phase difference histogram, the amplitude variance, and the frequency variance. The classification procedure as shown in Fig. 8 comprises $t \mathrm{e}$ following steps: 1) approximate signal bandwidth estimation, 2) signal demodulation and param ters extraction, 3) statistical computation, and 4) automation of modulation classification. In [1] it is clear that tre hardware implementation of this recognizer is excessively complex. In [1], it is claimed that an error free signal, i.e. all the signal parameters such as the carrier frequency, sym sol rate, ... etc. are exactly known, can be recognized at SNR $>18 \mathrm{~dB}$.

In [6] DeSimio and Glenn introduced an adaptive technique for classifying sorne types of digital modulations - ASK2, PSK2, PSK4 and FSK2. In this recognizer a set of key fealures derived from the signal envelope, the signal spectra, the signal squared and the fourth power of the signal are used to decide about the modulation type of the intercepted signal. These $k$ :y features are the mean and variance of the envelope, the tacetion of the peaks in the signal spectrum, the location of 
the peaks around twice the carrier frequency of the spectrum of the signal square, and the location of the peaks around four time the carrier frequency of the spectrum of the signal raised to the fourth power. In [6], the classification procedure consists of the following steps: 1) feature vectors extraction, 2) weight vectors generation for each signal class and 3) modulation classification. In [6], the decision functions used are generated using an adaptive technique based on the LMS algorithm. Furthermore, the decision rule used is similar to that applied in the pattern recognition algorithms. So, any intercepting signal is divided into two sets: a learning set, which is used to perform the weight vectors and a test set that is used in the decision about the modulation type using the weight vectors generated from the learning set. This classifier is trained using the values of the extracted key features at $20 \mathrm{~dB}$ SNR. The only thing mentioned about the performance evaluation of this recognizer is its ability to discriminate between PSK2 and PSK4 at the SNR of 5 dB.

Polydoros and Kim [10] introduced a modulation recognizer, following the decision-theoretic approach, to discriminate between PSK2 and PSK4. In [10], all signal parameters such as the carrier frequency, the initial phase, the symbol rate and the signal-to-noise ratio are assumed to be available. This recognizer uses the log-likelihood ratio to estimate the number of levels, $M$, of the MPSK signals. Also, a comparison between three classifiers for MPSK signals had been introduced. These classifiers are: 1) phase-based classifier (PBC) that is based on the phase difference histogram, 2) square-law classifier (SLC) that is based on the fact that squaring of MPSK signal is MPSK with M/2 phase states, and 3) quasi-log-likelihood ratio (QLLR) classifier which uses the likelihood ratio estimation principles. In [10], it is proved analytically that the performance of the QLLR classifier is significantly better than the PBC or the conventional SLC. Also, it is claimed that the QLLR classifier can be extended to address MPSK signals classification with $\mathrm{M}>4$.

Hsue and Soliman [11] introduced a modulation recognizer based only on the zero-crossings characteristic of the intercepted signals. The modulation classification procedure comprises three steps: 1) extraction of the zero-crossing sequence, the zero-crossing interval sequence and the zero-crossing interval difference sequence, 2) inter-symbol transition (IST) detection as well as carrier frequency estimation and 3 ) decision about the modulation type. The phase and frequency information are derived from the zero-crossing sequence, the zero-crossing difference sequence and the zero-crossing interval difference sequence. The decision about the modulation type is based on the variance of the zero-crossing interval sequence, $G$, as well as the frequency and phase difference histograms. This recognizer can be implemented using a parallel processing technique to increase the speed of computations. Also, three processors are recommended, each processor associated to one of the above mentioned three sequences. However this recognizer is used to report the modulation type of constant amplitude signals such as CW, MPSK, MFSK. In this recognizer, the classification strategy as shown in Fig. 9 comprises two main steps; first discrimination of single-tone (CW and MPSK) from multi-tone (MFSK) signals, and secondly determination of the number of states $(\mathrm{M})$. In [11], the discrimination between the single-tone and the multi-tone is based on comparing the variance of the zero-crossing difference sequence in the non-weak intervals of a sigral with a suitable threshold. Tue determination of the number of states in single-tone signals is achieved by measuring the similarity of the normalized phase difference histogram. The determination of the number of states in multi-tone signals is based on the number of hills in the zero-crossing interval difference histogram. Finally, the performance of this recognizer were derived from 100 realizations for each modulation type of interest. From the simulation results it is claimed that a reasonable average probability of correct classification is achievable for SNR > $15 \mathrm{~dB}$. 
Also, Soliman and Hsue [13] introduced another modulation recognizer bised on the statistical moments of the intercepted signal phase. In this recognizer, the even order noments of the signal phase are used to estimate the number of levels, M, in MPSK signals. The classification prociedure comprises the following steps: 1) instantaneous phase extraction, 2) ven order mornents computation, 3) threshold comparison and 4) decision about the modulation t/pe. It is claimed that the second order moment is sufficient to discriminate the CW from the MIPSK. signals and, the eighth order moment is adequate to classify BPSK signals with reasonab e performance $a$ t low SNR. A.lso, it is claimed that the suggested classifier is better than the PBC ar $d$ the SLC.

Assaleh et al. [14] proposed a modulation recognizer for some types of dic ital modulations. The types that can be classified by this recognizer are: CW, PSK2, PSK4, FSK?, and FSK4. The key features used were derived from the averaged spectrum of the instantaneous frequency. These key features are the mean and the standard deviation of the averaged instantanec us frequency, and the height of the spikes in the differential instantaneous frequency. It is claimed that the perforrance evaluation of this recognizer was derived from 1000 realizations for each modulation type of interest. Also, it was found that the success rate of the modulation type of interest is $>99 .(1 \%$ at the SNR of $15 \mathrm{~dB}$. As this recognizer uses the averaging over $M$ successive segments, long ignal duration is required and hence this recognizer is mainly suitable for the off-li ne aralysis.

Nagy [16] introduced a suggested procedure for a modulation classifier of nultichannel systems. This classifier was accomplished by dividing the analyzed signal into individual components and each signal component is classified using a single tone classifier. Thatypes that have been classified by this recognizer are CW, ASK, PSK2, PSK4 and FSK2. The de'veloped classifier comprises two steps. First, detect and filter each signal component in the estimated amplitude spectrum, e.g. the FSK2 is considered as two correlated ASK2 signals. Second, compute the differential phase to discriminate between the different types of single hirmonic signal. In [16] the performance of the developed recognizer was derived from 100 realizations for each modulation type of interest. Finally, it is claimed that all the single-tone types (C.W, ASK2, PSK2 and PSK4) have been classified with success rate $>90.0 \%$ at $10 \mathrm{~dB}$ SNR ex cept the ASK2 $i=87.0$ $\%$ ).

Beidas and Weber [18] proposed a modulation recognizer for MFSK sigrals. This recognizer is based on the time-domain Higher-Order Correlations. This classifier is used to discriminate between the MFSK signals. This classifier comprises a bank of matched filter each of w ich is tuned to one of a prescribed frequency locations and a set of successive correlators. Also, it is based on comparing the log likelihood function with a suitable threshold to decide about the number of levels of MFSK signals. In [18], it is claimed that this recognizer immunes the imperfect knowledge of exact frequency locations.

Huang and Polydoros [19] introduced an algorithm for classifying the MPSK signals and it is based on the likelihood function of the instantaneous phase. This algorithın utilizes the de zisiontheoretic approach as the likelihood function of the instantaneous phas? is compared with a suitable threshold. This recognizer can be considered as a generalization of the modslation recognizer introduced in [10] because it can be used for $M>4$. In [20] it s claimed that the best performance for the known MPSK ctassifiers (PBC, SLC, and QLLR) can be obtained from the QLLR classifier. 
Yang and Soliman [22] modify the modulation recognizer, introduced in [13] that uses the statistical moments as already mentioned to estimate M in the MPSK signals. This modification is in the way of approximating the probability distribution function of the instantaneous phase. In [22], the Fourier series expansion is used for the exact phase distribution approximation instead of the Tikhonov probability density function which is used in [13]. In [22] it is claimed that the proposed algorithm offers a $2 \mathrm{~dB}$ reduction of the SNR required for $99.0 \%$ success rate and it offers simpler moment computations than [13]. In both [13] and [21], nothing is mentioned about the performance evaluation of these two recognizers.

Azzouz and Nandi [23] proposed a modulation recognizer for the digital modulation types up to 4-levels (ASK2, ASK4, PSK2, PSK4, FSK2, and FSK4). The key features used are derived from three qualifying parameters - the instantaneous amplitude, the instantaneous phase, and the instantaneous frequency of the signal under consideration. These key features are: 1) the maximum value of the spectral power density of the normalized-centered instantaneous amplitude, 2) the standard deviation of the absolute value of the centered no.a-linear component of the instantaneous phase in the non-weak intervals of a signal segment, 3 ) the standard deviation of the direct (not absolute) value of the centered non-linear component of the instantaneous phase, 4) the standard deviation of the absolute value of the normalized-centered instantaneous amplitude, and 5) the standard deviation of the absolute value of the normalized instantaneous frequency. A detailed pictorial representation for key features extraction from a signal segment is shown in Fig. 10 in the form of a flowchart. In this recognizer, the discrimination between the different digitally modulated signals is as shown in Fig. 11. In [23], all the digital modulation types of interest have been classified with success rate $>90.0 \%$ at the SNR of $10 \mathrm{~dB}$ except PSK $4(89.25 \%$ success rate). At the SNR of $20 \mathrm{~dB}$ all the modulation types of interest have been classified with success rate $>96.0 \%$. Furthermore, Azzouz and Nandi [26] introduced a modification for decision flow of the algorithm shown in Fig. 11. In [26], it is mentioned that, based on the aforementioned four key features, many algorithms can be developed according to the chosen decision flow. It was found that for one of the modified algorithms, the overall success rate is $>99.0 \%$ at the SNR of $10 \mathrm{~dB}$, and it is $100 \%$ at the SNR of $20 \mathrm{~dB}$. In [24] and [26] due to the simplicity of the both the key features extraction as shown in Fig. 4 and the decision rules as shown in [24; Figs. 3.4, C.5 and C.6] (simple logic functions), these algorithms can be used for on-line analysis. Moreover, Nandi and Azzouz proposed a modulation recognizer which utilizes the ANN approach [25]. It is based on a single hidden layer ANN and the same data set used in [24]. It was found that all the modulation types of interest have been classified with success rate $>93.0 \%$. at $10 \mathrm{~dB}$ SNR and with success rate $>97.0 \%$ at $20 \mathrm{~dB}$. In [27], Azzouz and Nandi introduced a double hidden layer ANN modulation recognition algorithm. Using the same data set in [23] and [25], it was found that all the modulation types of interest have been classified with success rate $>96.0 \%$. except FSK2 $(=92.5 \%)$ at the SNR of $10 \mathrm{~dB}$ but at SNR of $20 \mathrm{~dB}$, the success rate is $>99.0 \%$.

\section{Recognizers for Both Analog and Digital Modulations (ADMRAs)}

In [2] Callaghan et al. proposed a modulation recognizer utilizing the envelope and the zerocrossings characteristics of the intercepted signal. This recognizer uses a phase-locked loop (PLL) for carrier recovery in the weak intervals of the intercepted signal segment. It is worth noting that in some modulation types such as DSB, MPSK and AM with high modulation depth, the carrier frequency may be severely suppressed or absent. Carrier recovery during the suppressed portions (weak intervals) is equivalent to receiving a signal with very low SNR. So, using the PLL as a carrier recovery (hardware solution) overcome the problem of needing high SNR for accurate 
instantaneous frequency estimation from the zero-crossings. The accuracy of this recognizer deteriorates rapidly if the receiver is not perfectly tuned to the center frequency. The modulation types that can be recognized by this recognizer are CW, AM, FM, FSK2. In [2, it is claimed that this recognizer requires $\mathrm{SNR}>20 \mathrm{~dB}$ for the correct recognition of the aforementioned modulation types.

Jondral [3] proposed a modulation recognizer utilizing the pattern recogniticn approach for the noise signal and two types of analog modulated signals - AM and SSB - as well as for four types of digitally modulated signals - ASK2, PSK2, FSK2 and FSK4. The key feature S used are derived from the instantaneous amplitude, phase and frequency. These key features ar: the instantanecus amplitude, phase difference and frequency histograms. In this classifier, the instantanecus amplitude histogram is computed for the normalized instantaneous amplitude. The cormalization is done with respect to the maximum values of the intercepted signal. As this classifier uses the pattern recognition approach, the received signal is divided into two adjacent sels: learning set and test set. The segment length used in this recognizer is 4096 samples for each riodulation type. In [3] real signals have been used and it is claimed that all the above mentioner! modulation types have been classified with success rate $>90.0 \%$ except the SSB $(=8.3 .0 \%)$ and FSK4 $(:=88.0 \%)$.

Aisbett in [5] proposed a modulation recognizer, based on some key features derived from the instantaneous amplitude and the instantaneous frequency of a signal. In [5], i: is mentioned that the developed key features are noise resistant, and they are $\mathrm{A}^{2}, \mathrm{AA}^{\prime}$ and $\mathrm{A}^{2}$ ' where $\mathrm{A}$ is the signal envelope, $A$ ' is the signal envelope derivative and 'is the instantaner us frequency. The estimation of these key features is derived from 10 realizations, each with 9) msec. length, "or each modulation type of interest. Also, another unbiased key feature is added in [5] which is the variance of the squared instantaneous amplitude minus its squared mean. Th: modulation typies that can be classified by this recognizer are: AM, DSB, FM, ASK2, PSK2, FSK:, and CW signals. In [5], it is claimed that the success rate of the discrimination between th: morlulation types appears to be good at least for strong SNRs.

Petrovic et al. [8] suggested a modulation recognizer based on the variations and the zerocrossing rate of the AM detector output as well as the variations in the FM d tector output. The modulation types that can be classified by this recognizer are AM, FM, SSB, CW, ASK2, FSK2. This modulation recognizer comprises three main steps as shown in Fig. 11. The se are: 1) AM and FM demodulation, 2) Key features extraction, and 3) modulation classification. Thre e key features are derived from the AM detector output and they are: detect the presence of the signal, measure the amplitude variations and measure the instantaneous amplitude zero-crossing, rate. Also for the FM detector output, a narrow band and a wide band FM detection are perform ed. The only thing that is mentioned about the performance evaluation is that the results of the pr liminary test with real signals show the success of this recognizer.

Martin [9] proposed a modulation recognizer for some analog and digital modu ation types. These types are: AM, FM, SSB, CW, ASK2, and FSK2. The key features used at ederived from the: instantaneous amplitude, the IF signal spectrum and its derivative. These kty features are: the amplitude histogram, the signal bandwidth, and the relationship between the sp:ctral components In [9] real signals have been used and it is claimed that all the modulation typ es of interest have been classified with success rate $>90.0 \%$ except the FM $(=80.0 \%)$.

Dominguez et al. [12] introduced a modulation recognizer which is a general approach for both analog and digital modulations. This recognizer is concerned with some types of analog and 
digitally modulated signals. These types are AM, DSB, SSB, FM, CW, noise and digitally modulated signals up to 4-levels. This recognizer comprises three subsystems: 1) pre-analysis subsystem, 2) features extraction subsystem and 3) classifier subsystem. The recognition algorithm is based on the histograms of the instantaneous amplitude, phase and frequency. In [12], it is claimed that this recognizer performed well and all types have been correctly classified at SNR $>40 \mathrm{~dB}$. At the SNR of $10 \mathrm{~dB}$ the probability of correct modulation recognition is $0 \%$ for all digital modulation types except for PSK4 (7.0\%) and at $15 \mathrm{~dB}$ SNR the performance is still wanting especially for FSK4 (56.0\%), FSK2 (84.0\%) and ASK4 (87.0\%). In [12] the number of samples per segment used in the performance evaluations is 3000 samples. However, it should be noted that this work attempts to identify most of the well known analog and digital modulation types.

Nandi and Azzouz in [26] developed a modulation recognizer for the analog modulation types considered in [22] as well as the digital modulations types considered in [23]. The four key features used in [22] are also used for this recognizer. Two of the key features used in [23] - the standard deviation of the absolute value of the normalized instantaneous amplitude and frequency are also used in this algorithm. Three other key features are introduced. These features are: 1) the standard deviation of the normalized-centered instantaneous amplitude in the non-weak intervals of a signal segment, 2) the kurtosis of the normalized instantaneous amplitude, and 3) the kurtosis of the normalized instantaneous frequency. A detailed pictorial representation for the key features extraction from an RF signal is shown in Fig. 12 in the form of a flowchart. The discrimination between the different analog and digital modulation types are carried out according to the procedure introduced in Fig. 13. In this recognizer, it was found that all the modulation types of interest have been classified with sucsess rate $>90.0 \%$ at the SNR of $15 \mathrm{~dB}$, except AM (= $88.8 \%)$, ASK4 $(=77.3 \%)$, and FSK4 $(=88.0 \%)$. Also, in $[26]$, an alternative view for the decision flow algorithm presented in Fig. 13 is introduced. Based on the aforementioned nine key features, many algorithms can be generated according to the sequence of applying these key features in the classification algorithm. The performance evaluations of the algorithms for the recognition of analog and digital modulations were derived from 400 realizations for tweleve analog modulated signals (as in [22]) as well as the six digital modulated signals (as in [23]). In [26], it was found that the overall success rate is $>93.0 \%$ at the SNR of $15 \mathrm{~dB}$. Finally, Azzouz and Nandi [27] introduced a modulation recognizer which utilizes the ANN approach. It was found that the overall success rate of this recognizer is $>96.0 \%$ at the SNR of $15 \mathrm{~dB}$.

\section{Comparisons of the reviewed modulation recognizers}

From the presentation of the aforementioned modulation recognizers (section II, III, and IV), we fined that some of these recognizers utilize the decision theoretic approach, some utilize the pattern recognition approach, some utilize the ANNs approach and others utilize combination of these approaches. Table I gives a comparison between the modulation recognizers used for analog modulated signals only. The table indicates the approach used in each recognizer. It also provides the weak and strong points of each recognizer. Table II and III do the same thing for DMRAs and ADMRAs respectively. 
Proceeding of the $1^{\text {st }}$ ICEENG conference, 24-26 March, 1998.

$5 P-1383$

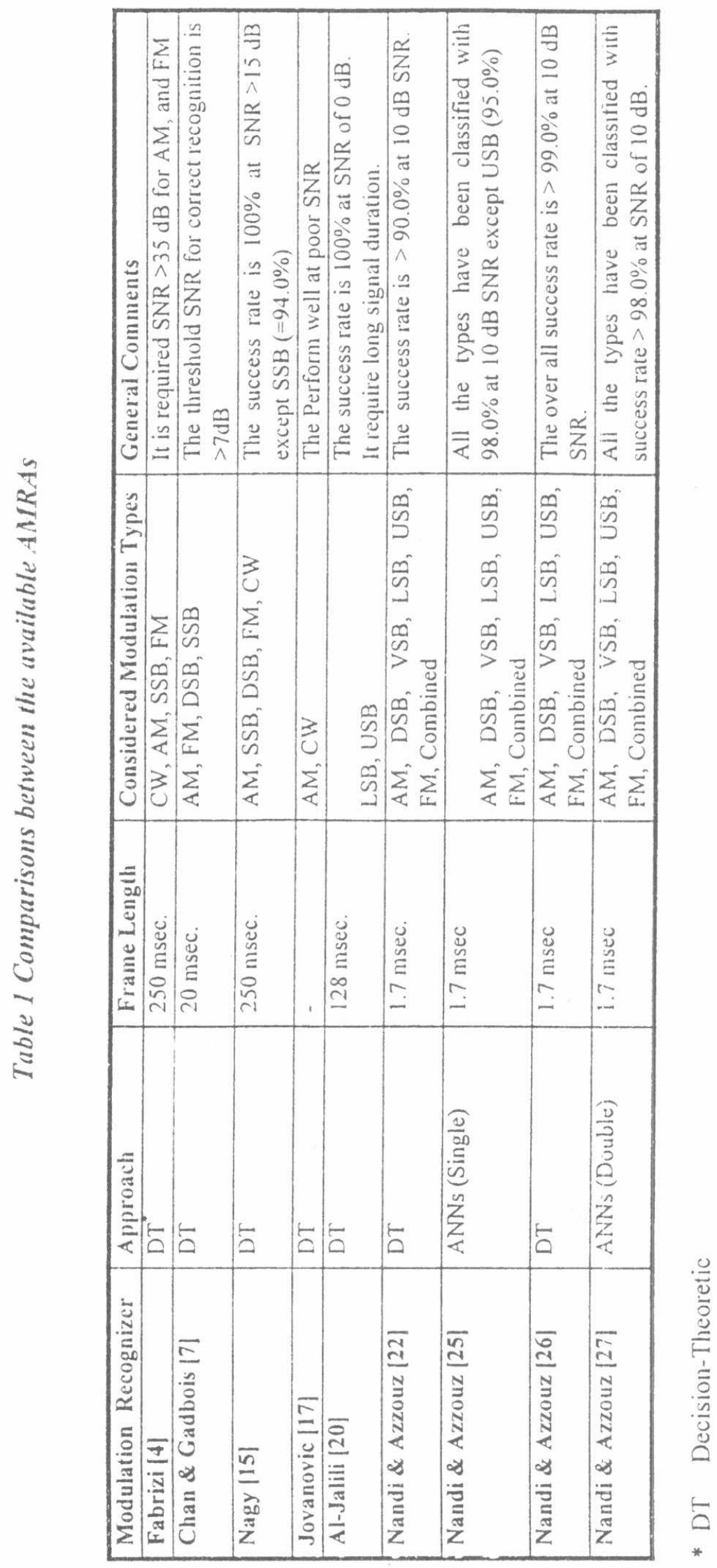


Proceeding of the $1^{\text {st }}$ ICEENG conference, 24-26 March, 1998.

$S P-139$

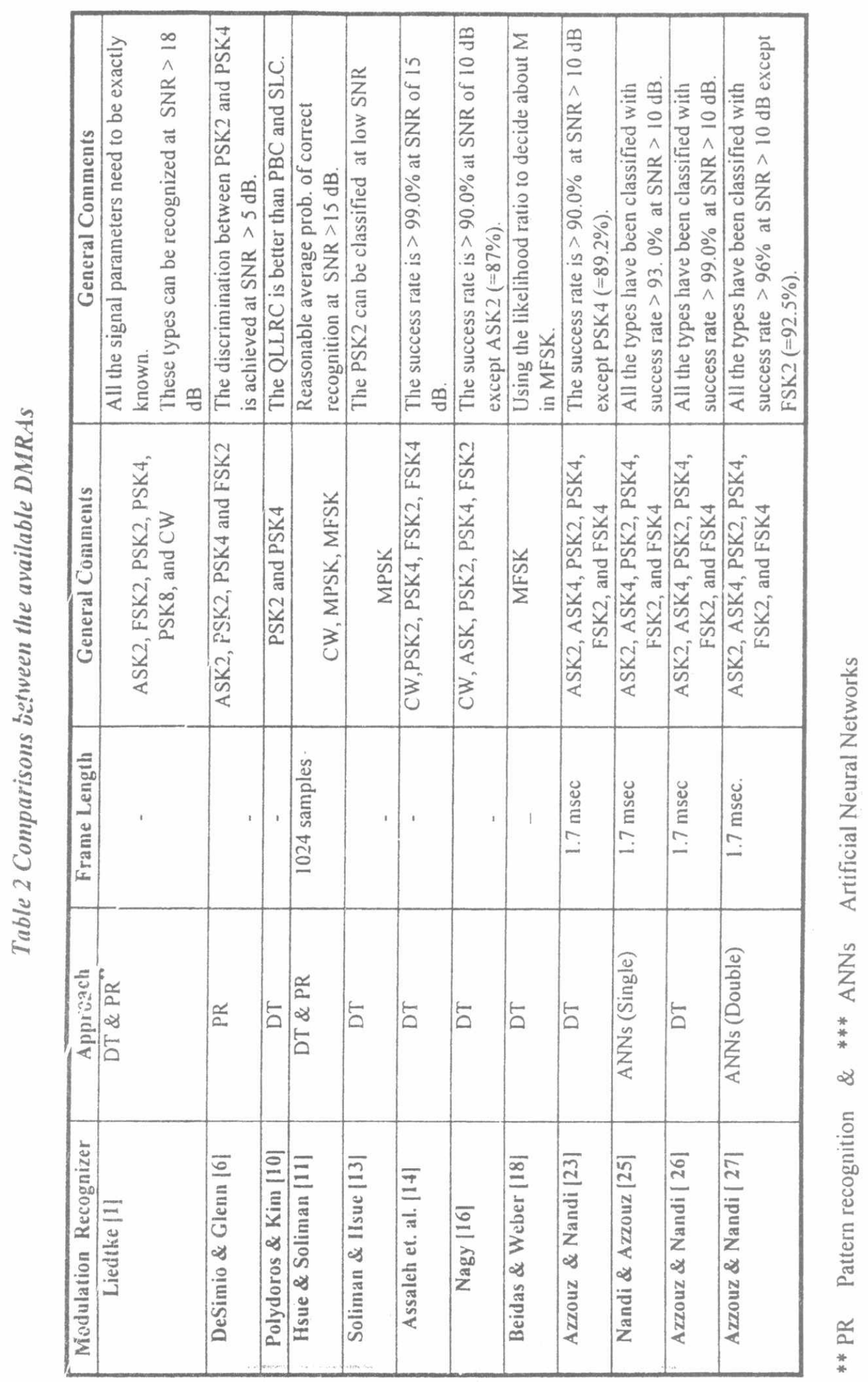


Proceeding of the $1^{\text {st }}$ ICEENG conference, 24-26 Miarch, 1998

$5 P-140$

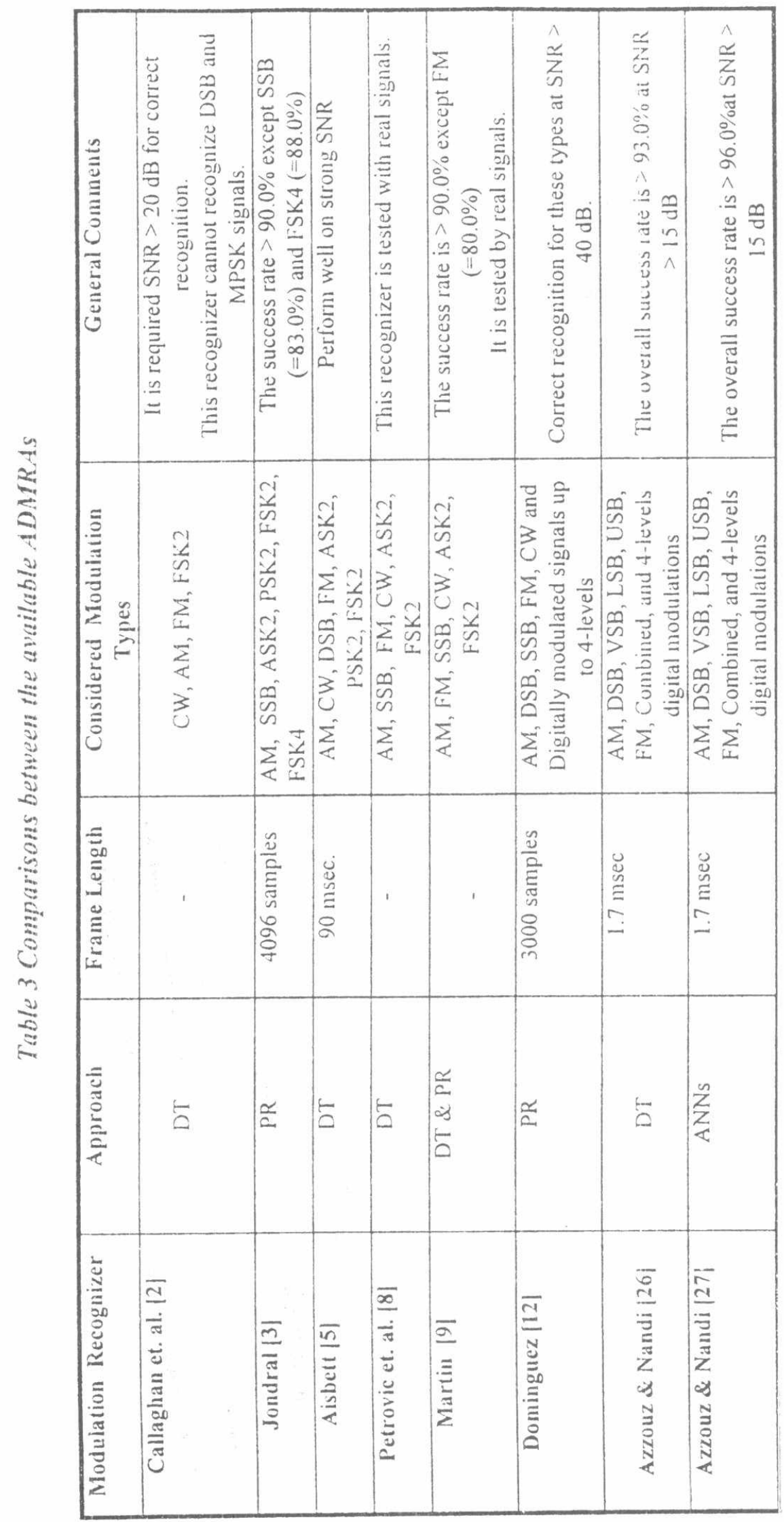


Proceeding of the $1^{\text {st }}$ ICEENG conference, 24-26 March, 1998.

\section{Open Issues and Proposals for Solutions}

While the work of Azzouz and Nandi [22-27] have treated many of the shortcomings found in the previous papers in the domain, they still have some shortcomings that need further investigations. In this section, we discuss these shortcomings and provide outlines of possible solutions. The first shortcoming is that assume exact knowledge of the carrier frequency. In practice, this assumption is not valid. Indeed, the carrier frequency of the intercepted signal is unknown and has some instability. This instability will create a noisy component in the instantaneous phase of the intercepted signal. Consequently, signals having amplitude modulation only may be erroneously classified as signals having both amplitude and phase information. To overcome this problem, we propose the use of one or more of the three following solutions: (1) on-line estimation of the carrier frequency, (2) increasing the phase threshold used in isolating phase signals, and (3) using the higher order statistics to reduce the effect of the noisy phase component. The carrier frequency instability will create also the following problem. In [22] and [24-27], the spectrum symmetry about the assumed carrier frequency is used to discriminate between symmetric signals (such as AM, DSB and FM signals) and spectrum asymmetric signals (such as SSB and VSB signals). The carrier frequency instability will disturbs the spectrum symmetry about the assumed carrier frequency as depicted in Fig. 14 in which the spectrum of an AM signal is considered. According to this figure, the power of the spectrum portion to the right side of the assumed carrier is much higher than its left side since the carrier usually contains a large part of the total power. In such a case, the signal will be erroneously classified as an upper sideband signal. This problem will take place even when on-line estimation of the carrier frequency is used since there will be always some frequency estimation error. The great part of the power included in the carrier component will cause a significant spectrum asymmetry about the estimated carrier frequency even when the estimation error is small. This problem may be overcome by excluding a zone with an "appropriate" width centered at the assumed carrier, when examining the spectrum symmetry.

The second shortcoming is the use of high sampling frequency that is equal to eight times the carrier frequency. This results in big storage and computational requirements. This shortcoming may be overcomed by the use of band-pass sampling instead of the low-pass sampling used the previous algorithms. It may also be overcome by the use of key features that may require less storage and computation requirements such as those derived from the complex envelope of a signal.

The third shortcoming is that the simulations therein use sampling frequency that is synchronized with the carrier frequency. In practice, however, the carrier frequency is generated by the hostile transmitter whereas the sampling frequency is generated at the interception receiver. Consequently, the two frequencies are not synchronized. Therefore the simulations should be redone with the two frequencies being asynchronous with each other. 
Proceeding of the $1^{\text {st }}$ ICEENG conference, 24-26 March, 1998.

\section{Conclusions}

A survey for the most of the recent pape's published in the area of modılation recognition is introduced. Three group of algorithms - Jne for analog modulations only, he second for digital modulations only and the third for brih analog and digital modulation:, without any prior information about the nature of a signal, are presented. Most of these re:ogn zers deal with a subset of the well known modulation types and a few of them consider ed the most corr mon modulation types. In [22] and [23], welve analog modulated signals and si: digitally modulated ones have been carried out to measure the performance of the presented algcrithms. Comparisons between the presented recognizers in each group are presented. Also, some general comments about each recognizer are summarized in Tables I-III. Finally, proposals sol tions for most of the short comings in the available references are presented.

\section{References}

[1] Liedtke, F. F., "Computer simulation of an automatic classification pr scedire for digitally modulated communication signals with unknown parameters,' Signal Processirig, Vol. 6, No. 4, August 1984, pp. 311-323.

[2] Callaghan, T. G., Pery, J. L. and Tjho, J. K., "Sampling and algorihms aid modulation recognition," Microvaves RF, Vol. 24, No. 9, September 1985, pp. 117-119, 121.

[3] Jondral, F., " Autematic classification of high frequency signals," Signal Pocessing, Vol. 9, No. 3, October 1985, pp. 177-190.

[4] Fabrizi, P. M., Lopes, L. B., and Lockhart, G. B., " Receiver recognition of arlalog modulation types," IERE Conference on Radio Receiver and Associated Systems, Bar gor, Wales, 1986, pp. 135-140.

[5] Aisbett, J., " Automatic modulation recognition using time domair parameters," Signal Processing, Vol. 13, No. 3, October 1987, pp. 323-328.

6] DeSimio, M. P. and Glenn, E. P., "Adaptive generation of decision function is for classification of digitally modulated signals," NAECON, 1988, pp. 1010-1014.

77] Chan, Y. T. and Gadbois, L. G., "Identification of the modulation typ e of a signal," Signal Processing, Vol. 16, No. 2, February 1989, pp. 149-154.

8] Petrovic, P. M., Krsmanovic, Z. B. and Remenski, N. K., " An automatic ''HF signal classifier", Mediterranean Electrotechnical Conference, MELECON, 1989, pp. 385-387.

[9] Martin, A., " A signal analysis and classification strategy for imple nentation in an EW communication receiver," Fifth International Conf. on Radic Receiver anil Associated Systems, July 1990, pp. 222-226

[10]Polydoros, A. and Kim, K., " On the detection and classification of quadrature digital modulations in broad-band noise," IEEE Transaction on Communications, '/ol. 38, No. 8, A.ugust 1990, pp. 1199-1211.

[11]Hsue, Z. S. and Soliman, S. S., "Automatic modulation classification using zero-crossing," IEE Proceedings. Part F Radar signal process, Vol. 137, No. 6, December 1990, fp. 4ミ9-464.

[12]Dominguez, L. V., Borrallo, J. M., and Garcia, J. P., " A general app oach to the automatic classification of radio communication signals," Signal Processing, Vol. 22, No. 3, March 1991, pp. 239-250.

[13]Soliman, S. S. and Hsue, Z. S., " Signal classification using statisti al moments," IEEE Transactions on Communications, Vol. 40, No. 5, May 1992, pp. 908-916. 
Proceeding of the $1^{\text {st }}$ ICEENG conference, 24-26 Miarch, 1998.

[14]Alssaleh, K., Farrell, K., and Mammone, R. J., " A new method of modulation classification for digitally modulated signals," MELCOM 92, Communication, Fusing, Command, Control, and Intelligence, October 1992. pp. 30.5.1-30.5.5.

[15]Nagy, P. A. J.," Analysis of a method for classification of analog modulated radio signals", European Association for Signal Processing VII Conference 94, Edinburgh, Scotland, September 1994, pp. 1015-1018.

[16]Nagy, P. A. J., " A modulation classifier for multi-channel systems and multi-transmitter situations", MILCOM 1994 Conference, 1994.

[17]Jovanovic, S. D., Doroslovacki, M. I., and Dragosevic, M. V., " Recognition of low modulation index AM signals in additive Gaussian noise," European Association for Signal Processing V Conference, Edinburgh, Scotland, September 1994, pp. 1923-1926.

[18]Beidas, B. F., and Weber, C. L., " Higher-order correlation- based approach to modulation classification of digitally modulated signals," IEEE Journal on selected areas in communications, Vol. 13. No. 1, January 1995.

[19]Huang, C. Y., and Polydoros, A., " Likelihood method for MPSK modulation classification," IEEE Transaction on Communications, Vol. 43, No. 3, March 1995, pp. 1493-1504.

[20]Al-jalili, Y. O., "Identification algorithm for upper sideband and lower sideband SSB signals," Signal Processing, Vol. 42, No. 2, March 1995, pp. 207-213.

[21]Yang, Y., and Soliman, S. S., "An improved moment-based algorithm for signal classification," Signal Processing, Vol. 43, No. 3, May 1995, pp. 231-244.

[22]Nandi, A. K., and Azzouz, E. E., "Recognition of analog modulations," Signal Processing, Vol. 46, No. 2, October 1995, pp. 211-222.

[23]Azzouz , E. E., and Nandi, A. K., "Automatic identification of digital modulations," Signal Processing, Vol. 47, No. 1, November 1995, pp. 55-69.

[24]Azzouz, E. E., and Nandi, A. K., "Automatic modulation recognition of communication signals", Kluwer Academic Publishers, Dordrecht, Netherlands, 1996.

[25]Nandi , A. K., and Azzouz, E. E., "Modulation recognbition using artificial neural networks", Signal Processing, Vol. 56, No. , 1997, pp. 165-175.

[26]Azzouz, E. E., and Nandi, A. K., "Automatic Modulation Recognition - Part I" to be appeared in Journal of Frankline Institute.

[27]Azzouz, E. E., and Nandi, A. K., "Automatic Modulation Recognition - Part II" to be appeared in Journal of Frankline Institute. 


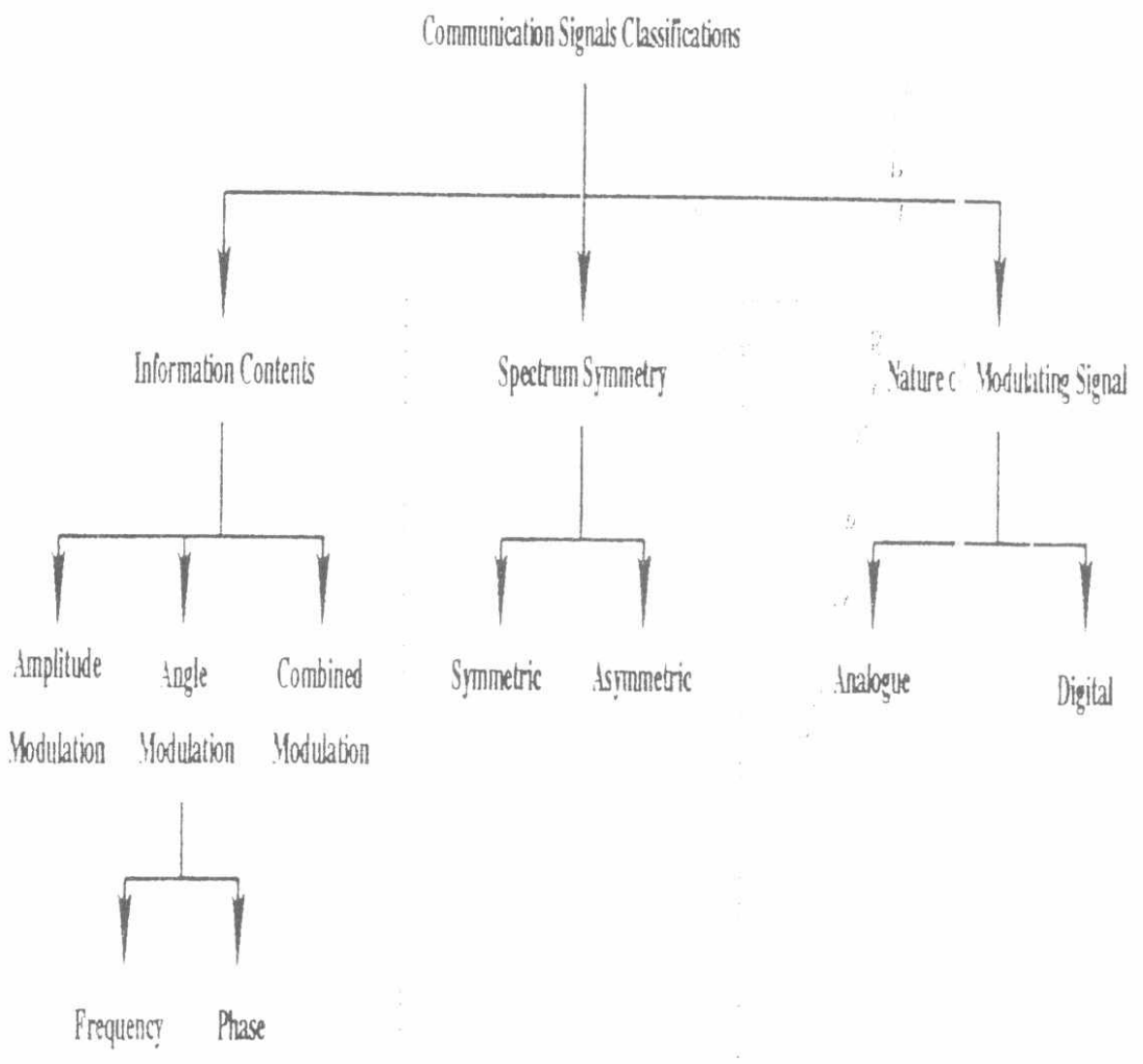

Fig. I Communication signals classifications

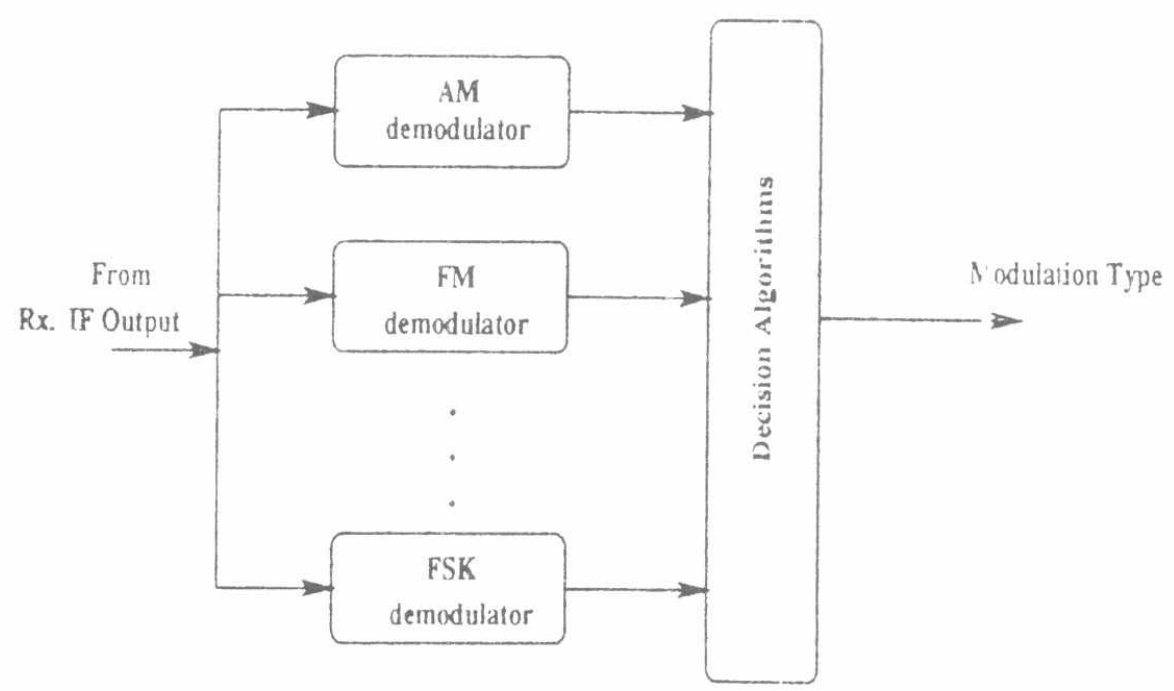

Fig. 2 A modulation recogniser using a bank of demodulators and a sety of decision algorithms. 


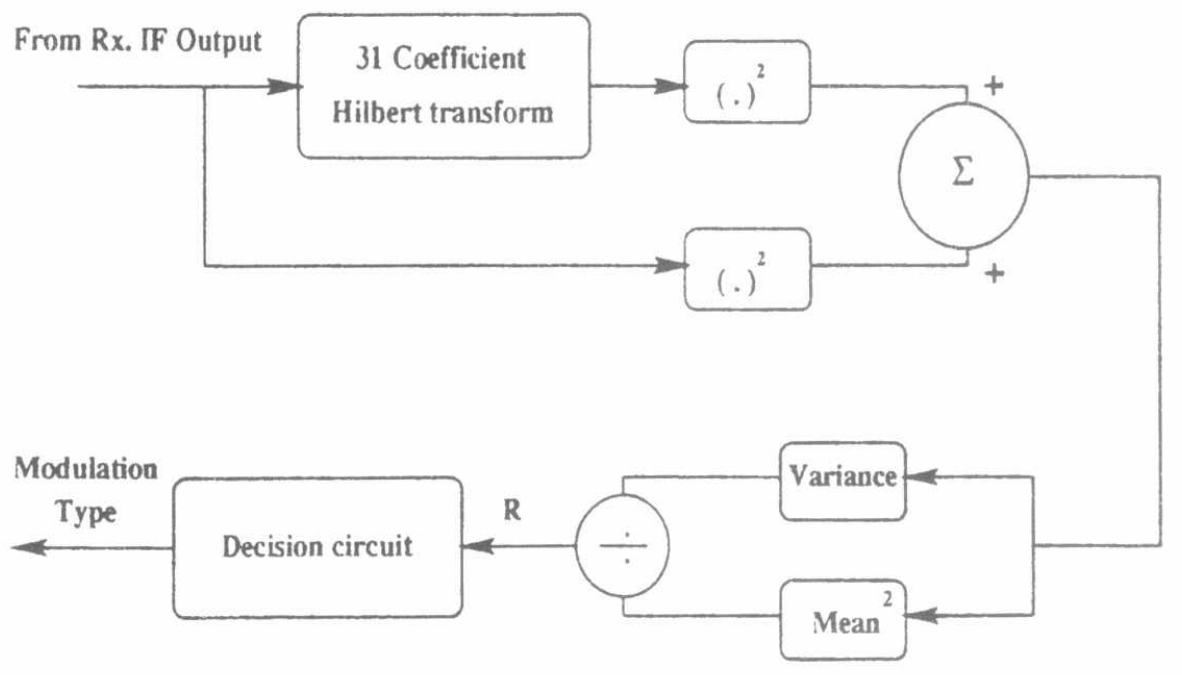

Fig. 3 Block scheme of the recogniser using the envolope characteristics only

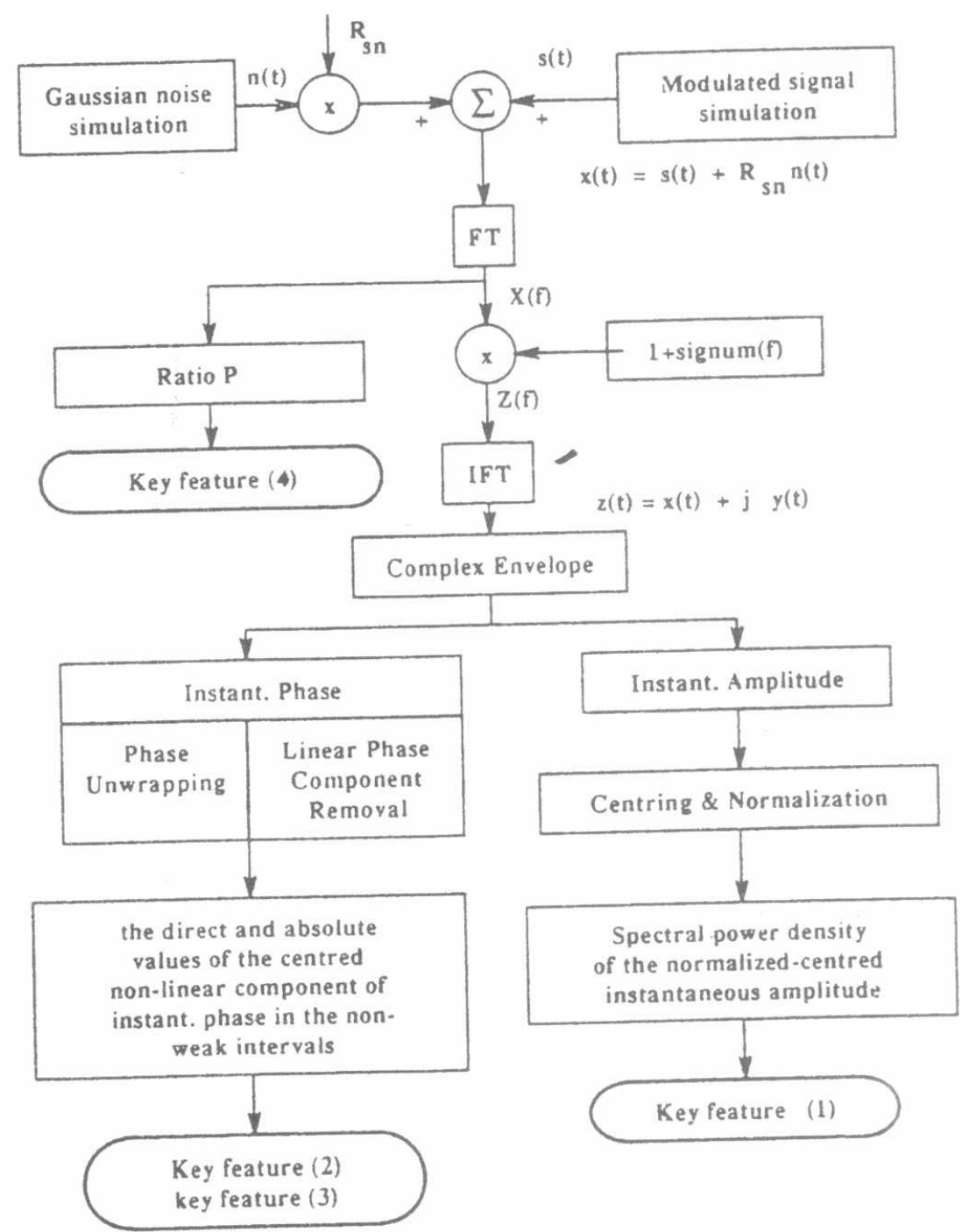

Fig. + Functional flowchart for key features extraction in the AMRAs [22] 


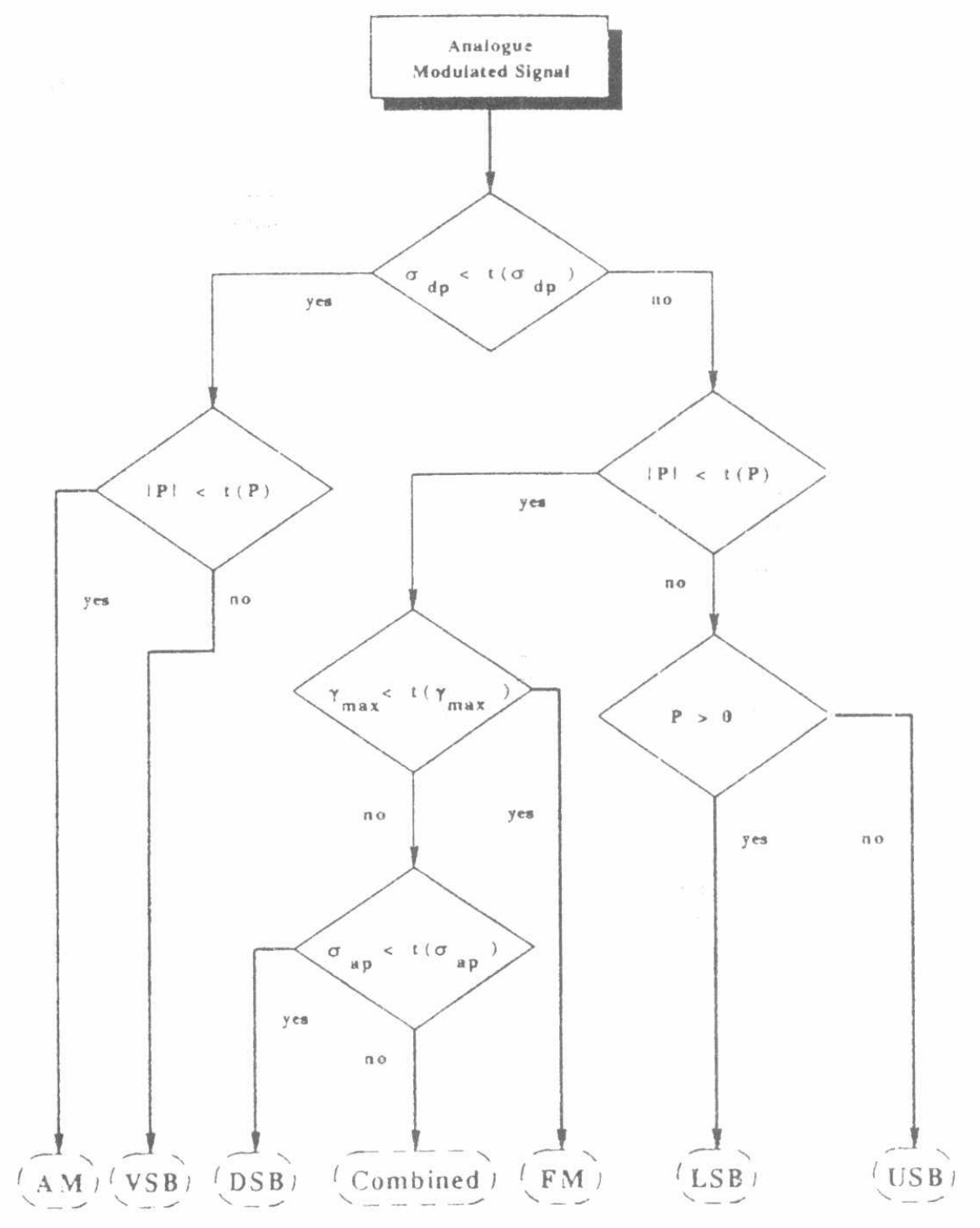

Fig. 5. Functional flowchart for AMRA [22] 
Proceeding of the $1^{\text {st }}$ ICEENG conference, 24-26 March, 1998.

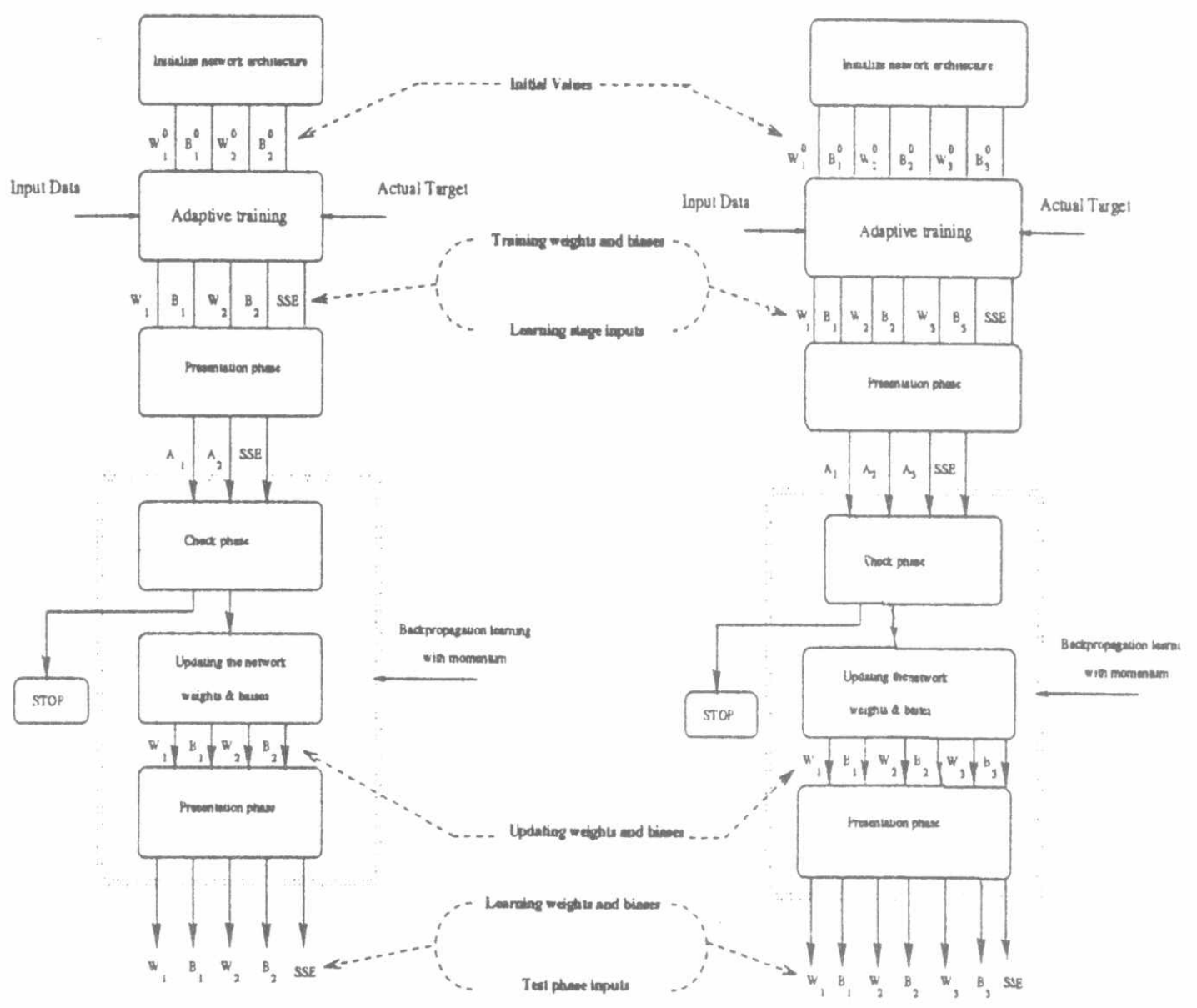

(a)

(b)

Fig. 6 Training procedure of the ANN algorithm: (a) single hidden layer, and (b) two hidden layer 
Weights and biases of the trained network

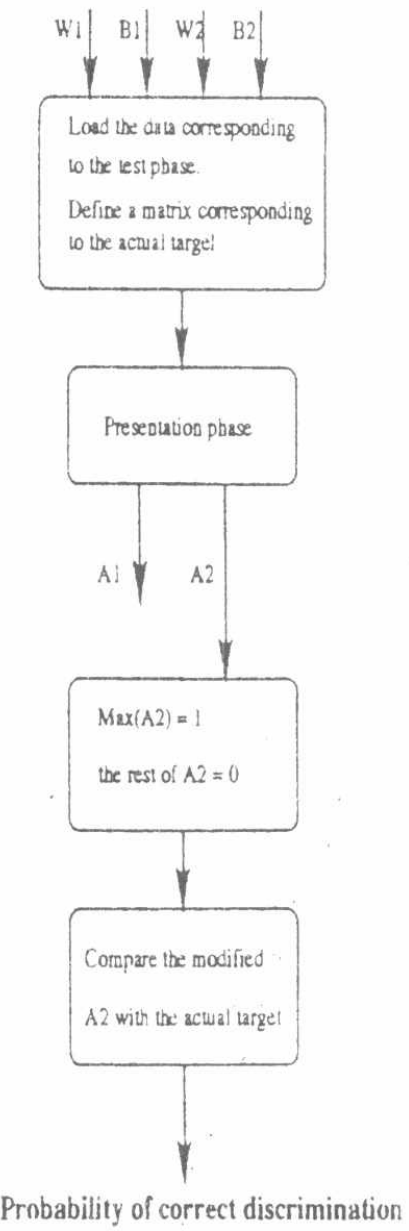

Weights and biases of th ? train:d network

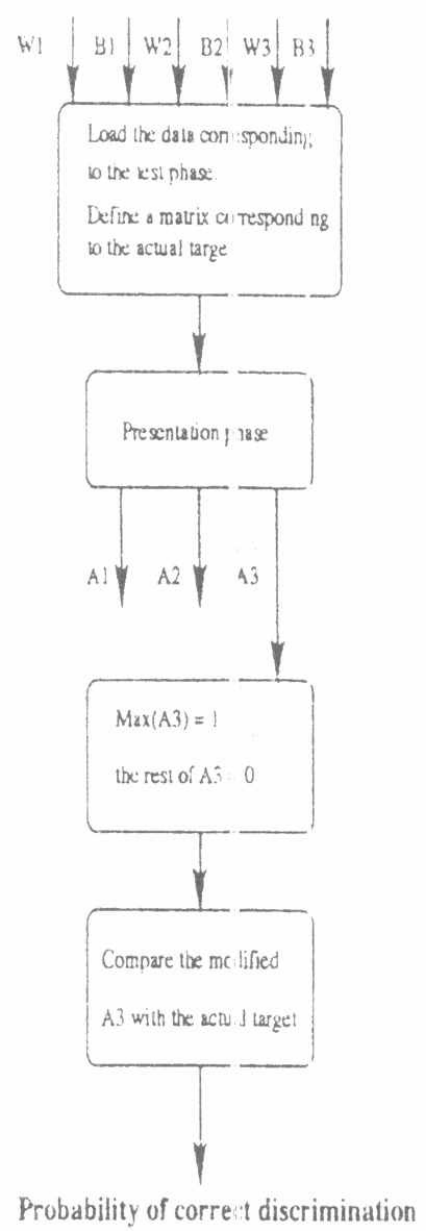

(a) 
Proceeding of the $1^{\text {st }}$ ICEENG conference, 24-26 March, 1998.

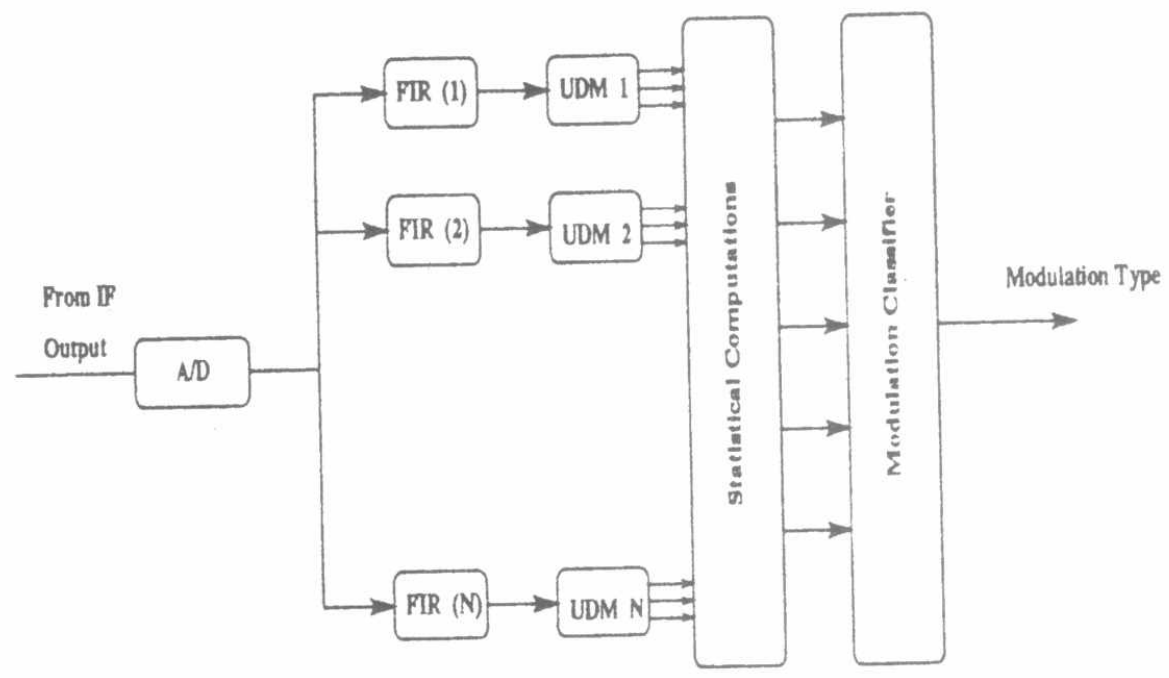

Fig. 8 Liedtke modulation recognizer [1]

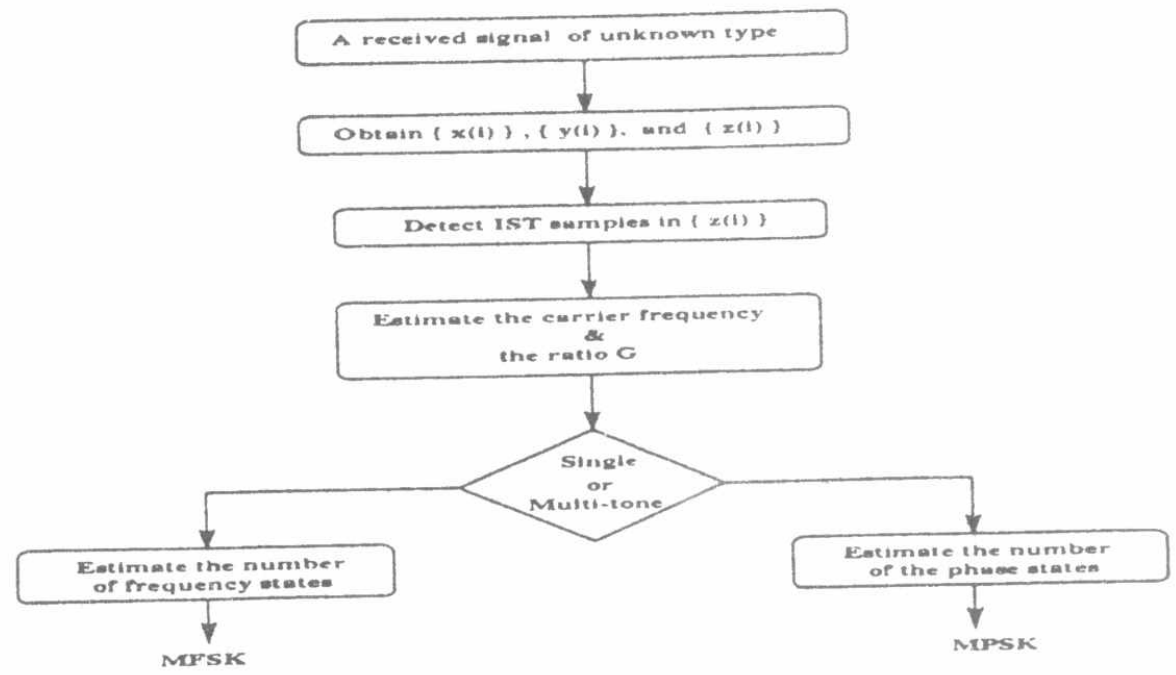

Fig. 9 Block scheme of a recognizer based on zero-crossings of a signal [23] 


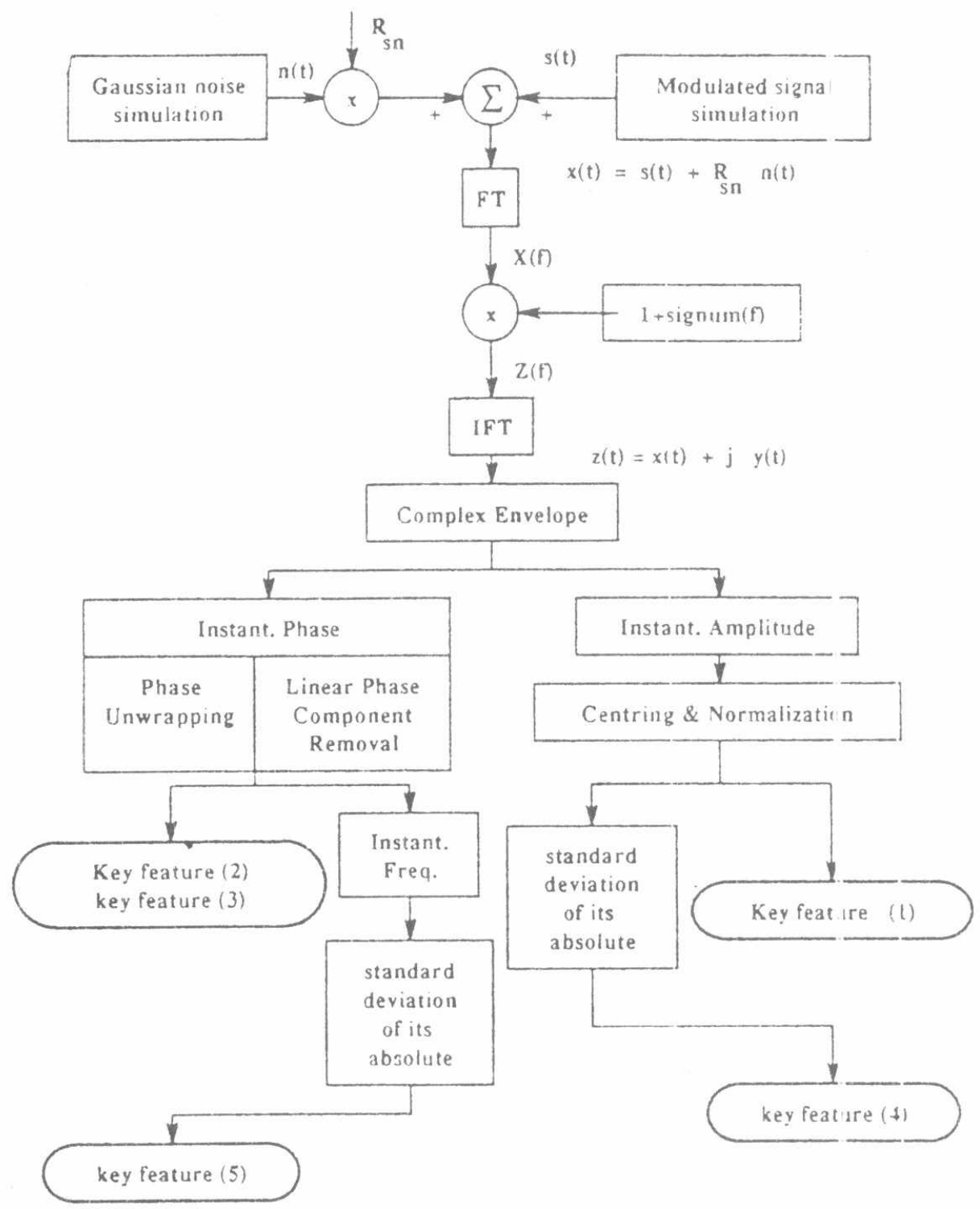

Fig. 10 Functional flowchart for kev features extraction in the DMRAs [23]. 


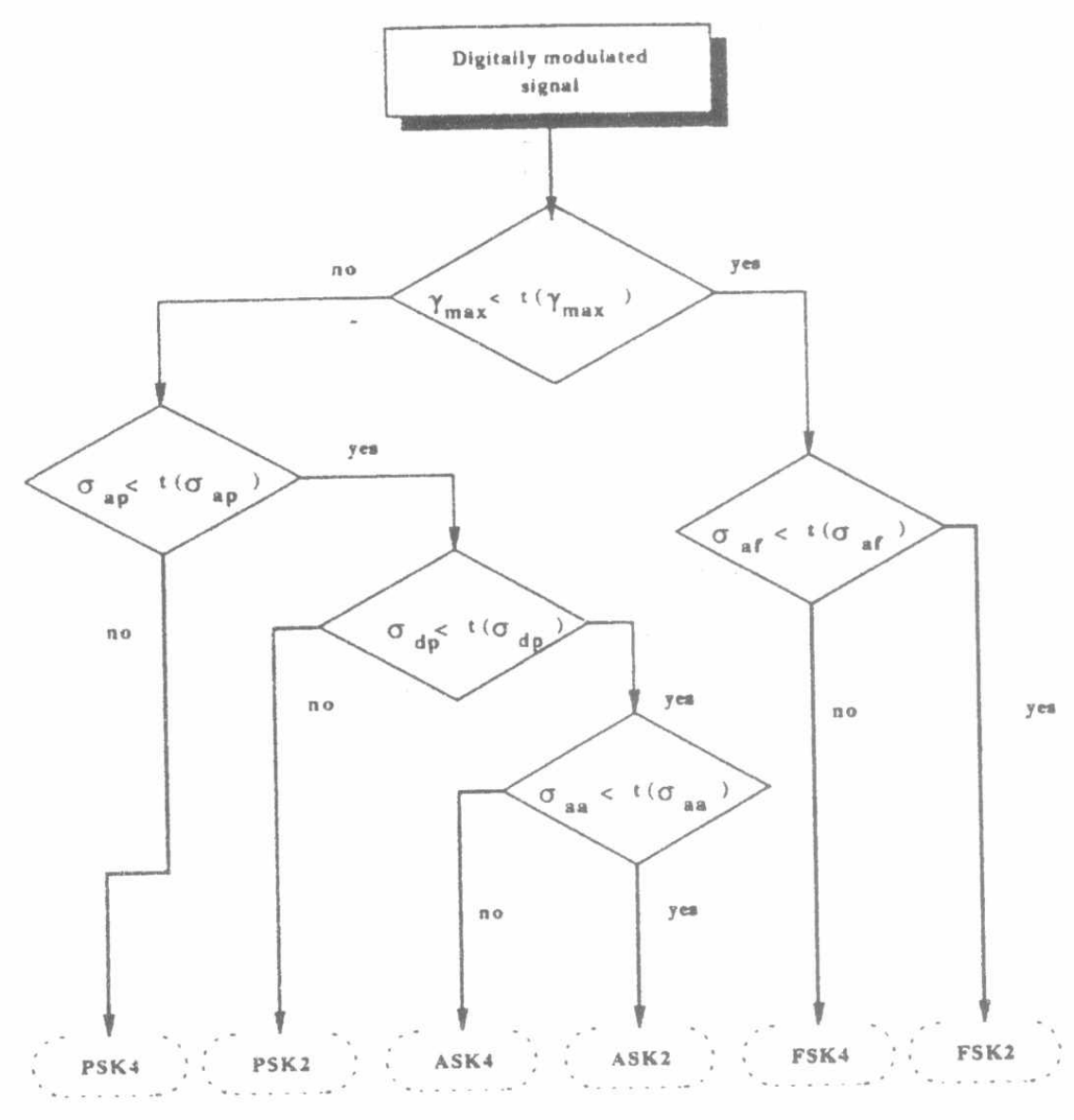

Fig. II Functional flowchart for DMRA [23] 


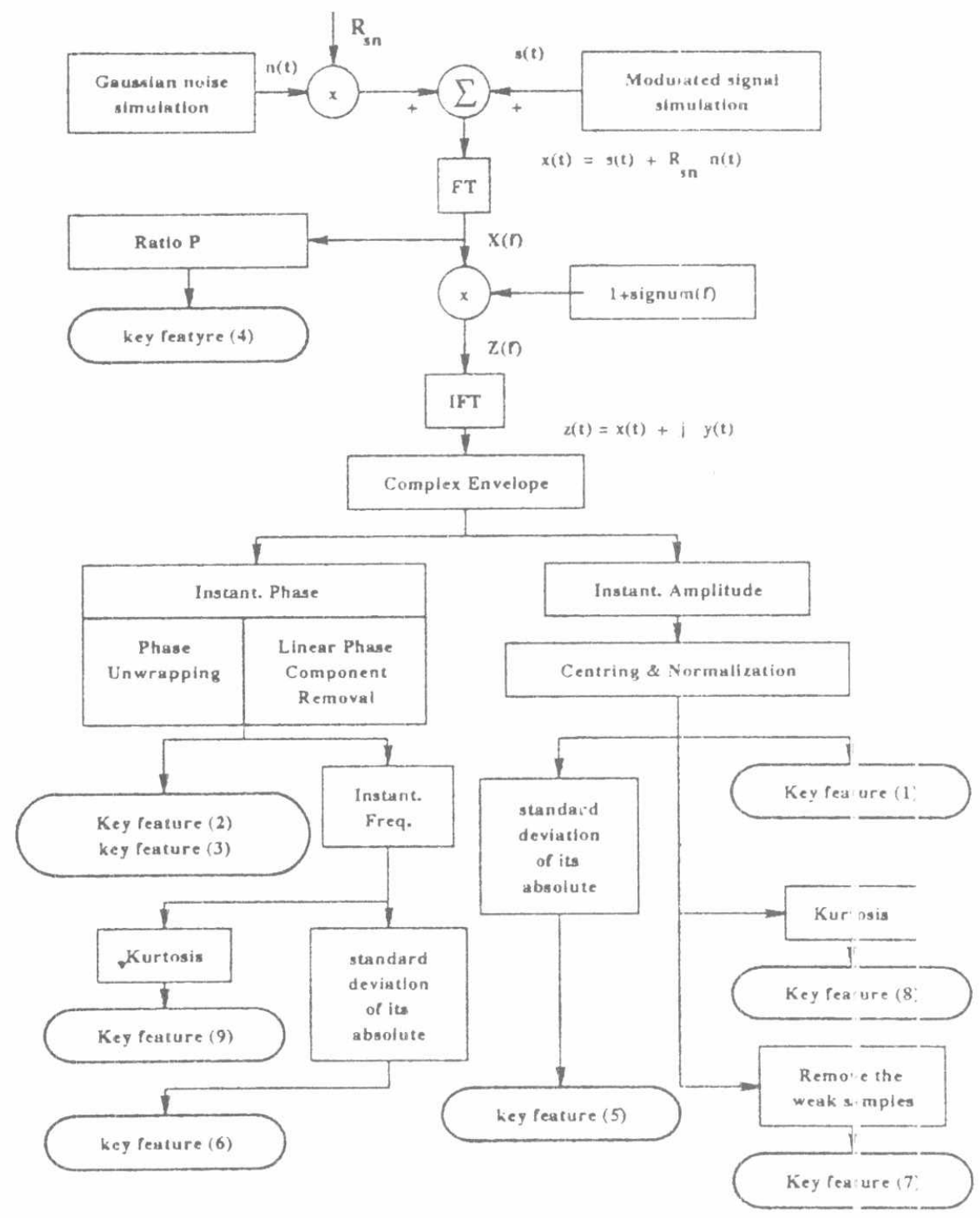

Fig. 12 Functional flowchart for key features extraction in the ADMRAs [26]. 
Proceeding of the $1^{\text {st }}$ ICEENG conference, 24-2 25 March, 1998.

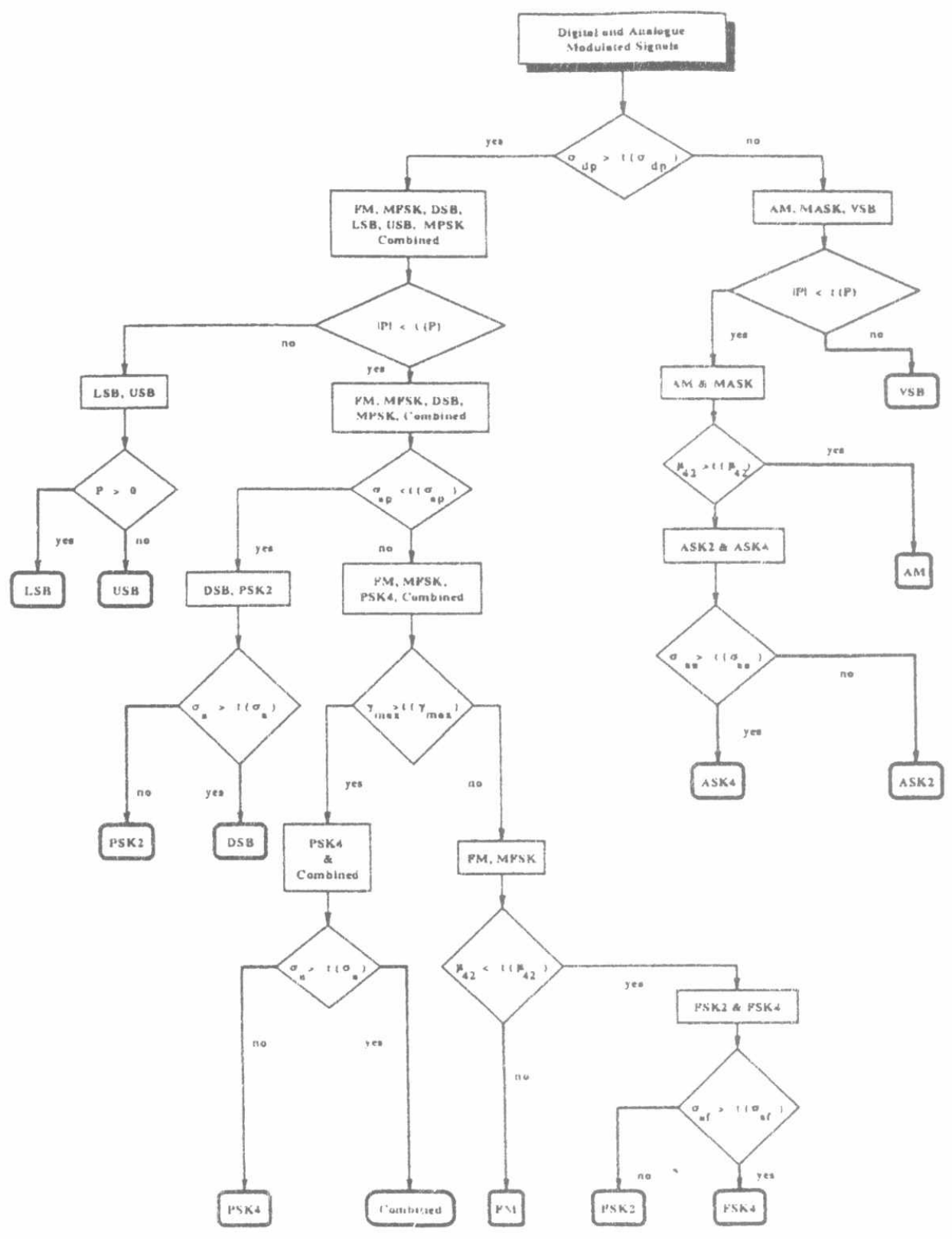

Fig. 13 Functional flowchart for ADMRA [26]

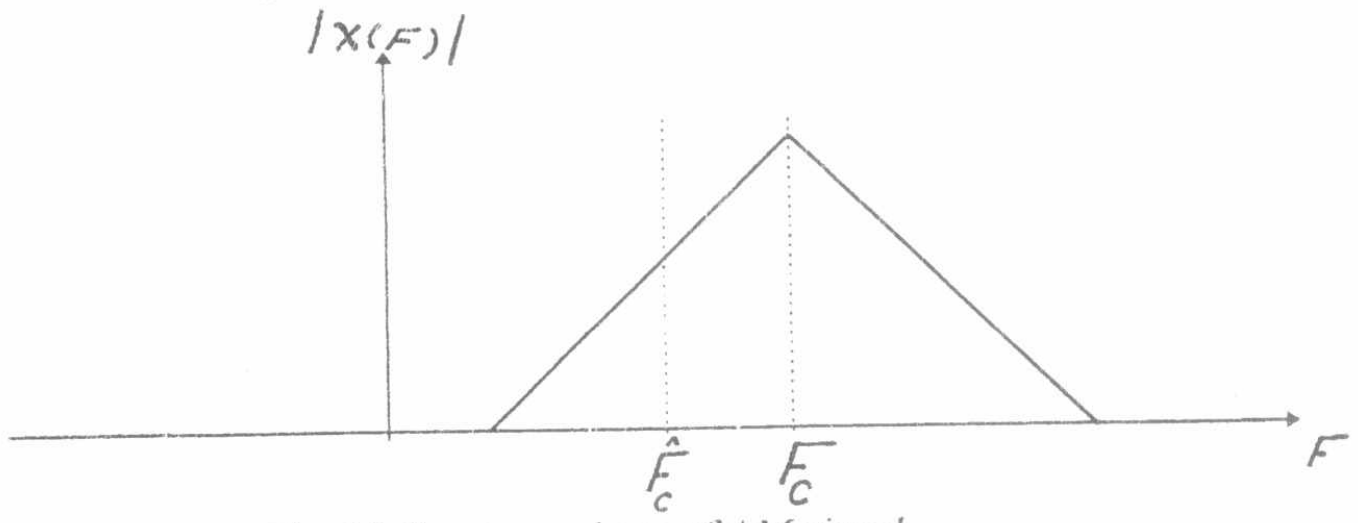

Fig. 14 Spectrum shape of AM signal 
